Artykuły

Studia Iuridica Lublinensia vol. XXVI, 1, 2017

DOI: 10.17951/sil.2017.26.1.519

José Ramón Sánchez Jaraba

Universidad de Jaén

ramonjaraba@hotmail.com

\title{
Las indicaciones geográficas agroalimentarias como motor de desarrollo de las economías territoriales
}

\author{
Chronione oznaczenia geograficzne produktów \\ rolno-żywnościowych jako impuls dla rozwoju \\ gospodarek krajowych
}

\section{RESUMEN}

La creciente comercialización de productos agroalimentarios elaborados en determinados territorios, cuya procedencia geográfica les confiere unas propiedades características especialmente valoradas por el consumidor a lo largo de varias generaciones, ha propiciado que la economía de numerosos territorios se base fundamentalmente en la producción y comercialización de este tipo de productos característicos, permitiendo la subsistencia e incluso prosperidad de muchas zonas que, en otras circunstancias, se hubieran visto avocadas al despoblamiento y a la desaparición. Por este motivo, entre otros, la protección de las indicaciones geográficas referidas a productos agroalimentarios ha constituido una importante preocupación por parte de los gobiernos nacionales y regionales, que ha propiciado el desarrollo de un importante régimen jurídico de protección. En este artículo se analizará el régimen jurídico a nivel de la Unión Europea para tutelar este tipo de referencias geográficas de calidad.

Palabras clave: propiedad industrial; Denominaciones de Origen Protegidas; Indicaciones Geográficas Protegidas; productos agroalimentarios; productos vinícolas; desarrollo territorial

\section{INTRODUCCIÓN}

La creciente importancia de las referencias geográficas de los productos agroalimentarios y su consolidación como motor de las economías de numerosos territorios ha propiciado que los estados y regiones pongan un especial interés en la salvaguarda de su integridad ante los ataques producidos por competidores desleales que quieren sacar partido a la buena reputación granjeada a lo largo de muchas generaciones. 
La Unión Europea ha querido desarrollar un régimen de protección adecuada para los productos agroalimentarios, estableciendo unas figuras de protección a nivel comunitario, las Denominaciones de Origen Protegidas (DOP) y las Indicaciones Geográficas Protegidas (IGP). En este artículo se analizarán los regímenes de protección que la Unión Europea ha establecido para los diferentes tipos de productos agroalimentarios, en torno a estas dos modalidades de referencias geográficas de calidad.

\section{OBJETIVOS Y MÉTODOS}

El objetivo principal de la investigación es dar a conocer y analizar los diferentes regímenes de protección de las referencias geográficas relacionadas con los productos agroalimentarios, proporcionando una visión general de los mismos que nos ayuden a tener una perspectiva amplia de la materia, sin ahondar en exceso en cada uno de ellos, comparándolos y estableciendo las principales diferencias y lo que cada uno de ellos aporta de novedad a la materia.

Se ha querido seguir, por tanto, un método descriptivo y analítico, que ponga en común todos los regímenes de protección y que nos proporcione una visión real de la materia objeto de estudio, dando a conocer las tendencias actuales y la posición que ocupa la misma en la mente del legislador comunitario.

\section{RESULTADOS DE LA INVESTIGACIÓN Y DISCUSIÓN.}

El Tratado constitutivo de la CE no contiene referencia alguna a las indicaciones geográficas, por lo que debemos acudir al derecho derivado para encontrar una regulación de las mismas, pudiendo diferenciar cuatro regímenes en función de los productos a considerar: productos vitivinícolas en general, productos vitivinícolas aromatizados, bebidas espirituosas y productos agroalimenticios en general.

Con carácter previo a la existencia de estos regímenes, la protección de las indicaciones geográficas en el ámbito comunitario se basaba en las distintas regulaciones nacionales de sus Estados miembros, cuyos derechos de protección exclusiva en ocasiones generaban conflictos con el principio de libre circulación de mercancías dentro del mercado único europeo. Efectivamente, el artículo 29 del Tratado constitutivo de la CE prohíbe las restricciones cuantitativas a la importación, así como todas las medidas de efecto equivalente, como contrarias al principio de la libre circulación de mercancías dentro del mercado único europeo. A primera vista, este artículo entra en controversia con el derecho que se confiere al titular de una indicación geográfica de prohibir las importaciones de productos con las mismas indicaciones dentro de su territorio. No obstante, el artículo 30 del citado Tratado, 
menciona entre las excepciones a la libre importación: las prohibiciones y restricciones justificadas por razones de protección de la propiedad industrial y comercial. Para determinar si las indicaciones geográficas se pueden encuadrar dentro de este tipo de propiedad, hay que acudir a la jurisprudencia comunitaria.

A este respecto, el Tribunal de Justicia de las Comunidades Europeas (TJCE) ha afirmado categóricamente que las denominaciones de origen forman parte de los derechos de propiedad industrial y comercial ${ }^{1} \mathrm{y}$, por tanto, en virtud del artículo $30 \mathrm{TCE}$, el artículo 29 del mismo, no es obstáculo para las prohibiciones o restricciones a la exportación justificadas, por razones de su protección ${ }^{2}$, en el caso de que impidieran a una denominación de origen cumplir con su función específica de garantizar que el producto amparado por la misma procede de una zona geográfica determinada y presenta determinados caracteres particulares ${ }^{3}$ y en la medida en que constituya un medio necesario y proporcionado para proteger la reputación de la $\mathrm{DOP}^{4}$.

Por tanto, la protección de este tipo de indicaciones geográficas, constituye en principio una excepción al principio de libre circulación de mercancías en la Unión Europea, con las matizaciones antes vistas: garantía de procedencia de una zona geográfica determinada, presencia de determinados caracteres particulares y aplicación de medios necesarios y proporcionales. Los dos primeros requisitos (garantizar en un producto la procedencia de una zona geográfica determinada y la presencia en el mismo de determinados caracteres particulares) son funciones específicas de las denominaciones de origen. Sin embargo, para valorar los criterios de necesidad y proporcionalidad de las medidas restrictivas adoptadas habría que acudir al caso concreto, teniendo en cuenta que:

[...] a efectos del examen de la proporcionalidad de la restricción de que se trata, es preciso comprobar aún si los medios aplicados en este contexto no van más allá de lo necesario para alcanzar el objetivo legítimo perseguido. En otros términos, será necesario valorar si no existen medidas alternativas que puedan realizar igualmente ese objetivo, pero con un efecto menos restrictivo sobre el comercio comunitario ${ }^{5}$.

Superada esta fase anterior sobre la admisión o no de la protección de las indicaciones geográficas por parte de la UE, el legislador comunitario ha regulado sobre dos grupos de productos bien diferenciados: los productos del sector vinícola, por un lado; y los productos agrícolas y alimenticios no vinícolas, por otro. Veamos su evolución hasta la situación actual.

\footnotetext{
${ }^{1}$ Sentencia del TJCE, de 16 de mayo de 2000, Bélgica/España, C-388/95, apartado 54.

${ }^{2}$ Sentencia del TJCE (Sala Primera), de 3 de marzo de 2011, recaída en el asunto C-161/09, apartado 36 .

${ }^{3}$ Sentencia de 9 de junio de 1992, dictada en el asunto C-47/90, apartados 16 y 17.

${ }^{4}$ Sentencia TJCE (Sala Primera), de 3 de marzo de 2011, recaída en el asunto C-161/09, apartado 37. Todas estas sentencias pueden consultarse en http://curia.europa.eu [accesso: 10.03.2017].

${ }^{5}$ Ibidem, apartado 39.
} 


\section{LAS INDICACIONES GEOGRÁFICAS DEL SECTOR VINÍCOLA EN EL MARCO COMUNITARIO}

En cuanto a los productos del sector vinícola, el Reglamento (CE) No 479/2008 del Consejo, de 29 de abril de 2008, estableció en materia de protección de denominaciones de origen protegidas e indicaciones geográficas protegidas un régimen equivalente al de la normativa comunitaria para los productos agrícolas y alimenticios no vinícolas, permitiendo que las mismas se reconocieran y registrasen a escala comunitaria. Este Reglamento fue derogado por el Reglamento (CE) No 491/2009 del Consejo, de 25 de mayo, aplicable a partir del 1 de agosto de 2009, el cual incorporó las normas del sector vitivinícola al denominado Reglamento único para las OCM [Reglamento (CE) No 1234/2007], al que modificó. Por su parte, este último, ha sido derogado por el Reglamento (UE) No 1308/2013 del Parlamento Europeo y del Consejo, por el que se crea la organización común de mercados de los productos agrarios.

Concretamente, la Sección 2, del Capítulo I, del Título II (artículos 92 y siguientes) del Reglamento 1308/2013 contiene la regulación relativa a las denominaciones de origen y las indicaciones geográficas en el sector vitivinícola, la cual solo es de aplicación a las siguientes categorías de productos vitícolas que se definen en el Anexo VII, parte II, del propio Reglamento: vino, vino de licor, vino espumoso, vino espumoso de calidad, vino espumoso aromático de calidad, vino de aguja, vino de aguja gasificado, mosto de uva parcialmente fermentado, vino de uvas pasificadas y vino de uvas sobremaduradas. No será de aplicación esta normativa sobre denominaciones de origen e indicaciones geográficas a estas otras categorías de productos vitícolas, igualmente definidas en el referido anexo: vino nuevo o en proceso de fermentación, vino espumoso gasificado, mosto de uva, mosto de uva parcialmente fermentado procedente de uva pasificada, mosto de uva concentrado, mosto de uva concentrado rectificado y vinagre de vino.

De acuerdo con la anterior normativa comunitaria sobre los productos vitivinícolas, contenida en el Reglamento 1493/1999, la competencia para reconocer y atribuir denominaciones de origen estaba reservada a los Estados miembros. La actual regulación atribuye a la Comisión Europea dicha competencia, tras superar un procedimiento nacional preliminar. Las fórmulas tradicionales de protección que se mencionaban en la anterior normativa, tales como Vinos de Calidad Producidos en Regiones Determinadas (VCPRD), vinos de mesa con indicación geográfica o Vinos Espumosos de Calidad Producidos en Regiones Determinadas, han quedado abandonadas en favor de las nuevas figuras de protección: Denominaciones de Origen Protegidas (DOP) e Indicaciones Geográficas Protegidas (IGP). Aunque existen diferencias entre estos dos tipos de figuras de protección, las líneas diferenciales entre las mismas no son tan claras como en las anteriores figuras de protección, donde los VCPRD, por ejemplo, constituían la figura que 
expresaba la calidad máxima de un vino, en contraposición a la categoría de los vinos de mesa. La nueva categorización de los vinos en DOP y IGP, amplía las fronteras de las áreas de calidad, incrementando el número de productos y productores que pueden verse beneficiados de una calidad, que anteriormente estaba reservada a pequeñas producciones y productores ${ }^{6}$.

El Reglamento 1308/20013, en su artículo 93, define como Denominación de Origen el nombre de una región, de un lugar determinado o, en casos excepcionales debidamente justificados ${ }^{7}$, de un país, que sirve para designar un producto vinícola de las categorías enunciadas anteriormente, que cumple los requisitos siguientes: 1) la calidad y las características del producto se deben básica o exclusivamente a un entorno geográfico particular, con los factores naturales y humanos inherentes a él; 2) las uvas utilizadas en la elaboración del producto proceden exclusivamente de esa zona geográfica; 3) la elaboración tiene lugar en esa zona geográfica; y 4) el producto se obtiene de variedades de vid de la especie Vitis vinifera.

Por otra parte, por Indicación Geográfica, según dicho artículo 93, se entenderá una indicación que se refiere a una región, a un lugar determinado o, en casos excepcionales debidamente justificados, a un país, que sirve para designar un producto vinícola de las categorías enunciadas anteriormente, que cumple los requisitos siguientes: 1) posee una calidad, una reputación u otras características específicas atribuibles a su origen geográfico; 2) al menos el $85 \%$ de la uva utilizada en su elaboración procede exclusivamente de esa zona geográfica; 3) la elaboración tiene lugar en esa zona geográfica; y 4) se obtiene de variedades de vid de la especie Vitis vinifera o de un cruce entre esta especie y otras especies del género Vitis.

Vistas estas definiciones, las diferencias entre ambas figuras de protección serían evidentes. La primera aparente diferencia sería una diferencia de tipo conceptual, ya que, como denominación de origen, solo podría considerarse el nombre de una zona geográfica, mientras que como indicación geográfica, cabría entender también otros signos distintos al nombre que hagan referencia a dicha zona. Para aclarar esto, el punto 2 del artículo 93 determina que ciertos nombres usados tradicionalmente constituirán una denominación de origen cuando designen un vino y cumpliendo el resto de requisitos de las denominaciones de origen y sometidos a sus procedimientos de protección establecidos, simplemente hagan referencia a un nombre geográfico. Por tanto, atendiendo a esta aclaración, podemos afirmar que no existe diferencia de tipo conceptual entre Denominaciones de Origen e Indicaciones Geográficas para designar productos vitivinícolas.

${ }^{6}$ F. Albisinni, Wines, [en:] European Food Law, Coord. L. Costato, F. Albisinni, Milán 2012, Capítulo XXV, p. 429.

${ }^{7}$ La expresión debidamente justificados no se contenía en la redacción anterior dada por el Reglamento 1234/2007, redundando así en la excepcionalidad que supone el nombre de un país como denominación de origen de un producto. 
La segunda y tercera diferencias hacen referencia a los requisitos de elaboración del producto que designan, siendo una diferencia de tipo cuantitativo, y otra, de tipo cualitativo. En este sentido, la materia prima utilizada para la elaboración del producto designado bajo la denominación de origen ha de proceder íntegramente de la zona geográfica en cuestión, mientras que en la indicación geográfica se permite que un determinado porcentaje (15\%) proceda de un lugar distinto (diferencia cuantitativa). Asimismo, en la elaboración del producto garantizado por una denominación de origen, solo pueden utilizarse vides de la especie Vitis vinifera, mientras que los productos amparados por una indicación geográfica se pueden obtener de vides de dicha especie cruzadas con otras del género Vitis (diferencia cualitativa) ${ }^{8}$.

El Reglamento amplía su campo de protección en la Unión Europea a las denominaciones de origen y a las indicaciones geográficas referentes a zonas geográficas de terceros países ${ }^{9}$ y establece un régimen de protección de los denominados términos tradicionales, como expresiones tradicionalmente empleadas en los Estados miembros para referirse a productos vinícolas acogidos a una denominación de origen o indicación geográfica protegidas y que se puedan caracterizar por el método elaboración, envejecimiento, calidad, color, tipo de lugar, $\mathrm{o}$ acontecimiento concreto vinculado a la historia del producto ${ }^{10}$.

\section{LAS INDICACIONES GEOGRÁFICAS DEL SECTOR NO VINÍCOLA EN EL MARCO COMUNITARIO}

En lo que se refiere a productos agrícolas y alimenticios no vinícolas, la protección comunitaria de las referencias geográficas viene regulada en el Reglamento (UE) 1151/2012 del Parlamento Europeo y del Consejo, de 21 de noviembre de 2012, sobre los regímenes de calidad de los productos agrícolas y alimenticios, el cual derogó la anterior normativa contenida en el Reglamento (CE) 510/2006 del Consejo, de 20 de marzo de 2006. Esta normativa recoge igualmente las dos figuras de protección antes descritas, indicaciones geográficas y denominaciones de origen, si bien su formulación, como se verá, es bien distinta.

El ámbito de aplicación de la protección de esta normativa abarca todos los productos agrícolas destinados a la alimentación humana contenidos en el Anexo I del Tratado CE y de los productos alimenticios y agrícolas siguientes: cerveza; chocolate y productos derivados; productos de panadería, pastelería, repostería y galletería; bebidas a base de extractos de plantas; pastas alimenticias; sal; gomas

${ }^{8}$ Conviene especificar que el mencionado 15 por ciento ajeno a la zona geográfica debe proceder del mismo estado de origen de la parte principal de uva (ver artículo 93.5 del Reglamento 1308/2013).

${ }^{9}$ Artículo 93.3 del Reglamento 1308/2013.

${ }^{10}$ Artículos 112 y 113 del Reglamento 1308/2013. 
y resinas naturales; pasta de mostaza; heno; aceites esenciales; corcho; cochinilla; flores y plantas ornamentales; algodón; lana; mimbre; lino espadillado; cuero; pieles, y plumas (según se relaciona en el Anexo I del propio Reglamento, listado que es susceptible de ampliación). Se excluyen los productos del sector vitivinícola, excepto el vinagre de vino, así como las bebidas espirituosas ${ }^{11}$.

A los efectos del citado Reglamento, se entenderá por «denominación de origen» un nombre que identifica un producto: 1) originario de un lugar determinado, una región o, excepcionalmente, un país; 2) cuya calidad o características se deben fundamental o exclusivamente a un medio geográfico particular, con los factores naturales y humanos inherentes a él; y 3 ) cuyas fases de producción tengan lugar en su totalidad en la zona geográfica definida.

Se entenderá por «indicación geográfica» un nombre que identifica un producto: 1) originario de un lugar determinado, una región o un país; 2) que posea una cualidad determinada, una reputación u otra característica que pueda esencialmente atribuirse a su origen geográfico; y 3) de cuyas fases de producción, una al menos tenga lugar en la zona geográfica definida ${ }^{12}$.

Como puede observarse, las definiciones de denominación de origen y de indicación geográfica contenidas en esta normativa se asemejan bastante. Como semejanza, ambas figuras vienen configuradas por el nombre que identifica un producto originario de una región, de un lugar determinado o de un país. Como diferencia, en primer lugar puede apreciarse una vinculación mayor al territorio de los productos amparados por una denominación de origen, toda vez que se exige que todas las fases de producción se realicen en la mencionada zona; mientras que para reconocer una indicación geográfica bastaría que alguna de sus fases de producción se hubiera efectuado en el lugar. Aparte de esta diferencia, existe otra diferencia fundamental relacionada con las cualidades o características del producto: en la denominación de origen estas cualidades o características han de deberse fundamental o exclusivamente a un medio geográfico particular con los factores naturales y humanos inherentes a él, mientras que para los productos protegidos por una indicación geográfica es suficiente que alguna de sus cualidades u otras características (incluida su reputación) pueda esencialmente atribuirse a dicho origen geográfico.

Al margen del contenido de estas definiciones, el Reglamento 1151/2012 asimila excepcionalmente a denominaciones de origen aquellas designaciones geográficas de productos cuyas materias primas estén sujetas a condiciones específicas y controladas de producción, pero procedan de una zona más amplia o diferente de la zona de transformación, siempre y cuando dichas designaciones hayan sido reconocidas como denominaciones de origen en sus países de origen antes del 1 de mayo de $2004^{13}$.

\footnotetext{
${ }^{11}$ Artículo 2 del Reglamento 1151/2012.

${ }_{12}$ Artículo 5 del citado Reglamento.

${ }^{13}$ Párrafo $3^{\circ}$ del artículo 5 del Reglamento 1151/2012.
} 
Si comparamos ambos sectores: vinícolas y no vinícolas, las definiciones de denominación de origen y de indicación geográfica no se diferencian tanto en este último sector. Conceptualmente, ambas figuras se refieren a un nombre, no cabiendo por tanto otros signos diferentes a éste, como sí vimos que podía ocurrir en las indicaciones geográficas garantizadoras de productos vitivinícolas. Cuantitativamente, tampoco existen diferencias, pues no se le exige a los productos amparados por una denominación de origen una vinculación mayor al territorio que a los amparados por una indicación geográfica, cuestión que no era así para los productos vinícolas, para los cuales se permitía que un 15 por ciento de la materia prima procediera de una zona distinta a la protegida por una indicación geográfica.

No obstante, sí parece existir una mayor vinculación entre producto y territorio en las denominaciones de origen, toda vez que se exige que todo el proceso de obtención del producto se realice en la mencionada zona (producción, transformación y elaboración), mientras que para reconocer una indicación geográfica bastaría que alguna de estas fases de obtención del mismo se hubiera efectuado en la zona. Aparte de esta diferencia, existe otra diferencia fundamental relacionada con las cualidades o características del producto. En la denominación de origen estas cualidades o características han de deberse fundamental o exclusivamente al medio geográfico con sus factores naturales y humanos, mientras que para los productos protegidos por una indicación geográfica es suficiente que sus cualidades u otras características (incluida su reputación) puedan atribuirse a dicho origen geográfico.

Con independencia de las Denominaciones de Origen e Indicaciones Geográficas Protegidas, el Reglamento 1151/2012, en sus artículos 17 a 26, establece un régimen de especialidades tradicionales garantizadas para proteger los métodos de producción y las recetas tradicionales, estableciendo un procedimiento de registro europeo de dichas especialidades ${ }^{14}$. Asimismo, en sus artículos 27 a 34, establece un segundo nivel de sistemas de calidad, con un régimen de términos de calidad facultativos para facilitar la comunicación de las características o atributos de los productos agrícolas que aporten valor añadido, estableciendo como tal el «producto de montaña» y proponiendo para su estudio el «producto de la agricultura insular», sobre el cual la Comisión emitió un informe en fecha 16 de diciembre de 2016, invitando al Parlamento y al Consejo a debatir sobre su conveniencia ${ }^{15}$.

${ }^{14}$ El derogado Reglamento 510/2006 asimilaba las denominaciones tradicionales a las indicaciones geográficas o denominaciones de origen, según correspondiera, aunque no fueran geográficas, siempre y cuando los productos agrícolas o alimenticios que amparasen cumplieran las condiciones para una u otra figura de protección.

${ }^{15}$ Informe de la Comisión al Parlamento Europeo y al Consejo sobre la Conveniencia del término de calidad facultativo ,producto de la agricultura insular”. Bruselas, 16.12.2013. COM(2013) 888 final. 


\section{OTRAS INDICACIONES GEOGRÁFICAS DE PRODUCTOS AGROALIMENTARIOS}

Como indicábamos en el apartado anterior, las bebidas espirituosas quedaban al margen de la regulación del Reglamento 1151/2012, como ya lo hacía de su predecesor Reglamento 510/2006. Pero dada la importancia de este tipo de productos en el tráfico económico europeo y la necesaria protección de los usuarios y consumidores, se hace necesario establecer un régimen de protección de las referencias geográficas de esta clase de productos. Este es el cometido del Reglamento (CE) $\mathrm{n}^{\mathrm{o}}$ 110/2008, del Parlamento Europeo y del Consejo, de 15 de enero de 2008, relativo a la definición, designación, presentación, etiquetado y protección de las indicaciones geográficas de bebidas espirituosas.

En su artículo 15, el Reglamento 110/2008 define "indicación geográfica" como "aquella que identifique a una bebida espirituosa como originaria del territorio de un país o de una región o localidad de ese territorio, si determinada calidad, reputación u otras características de la bebida espirituosa son imputables fundamentalmente a su origen geográfico", estableciendo en su Anexo III el listado de indicaciones geográficas que son objeto de protección al amparo de dicha normativa.

Por último, dado que el Reglamento 110/2008, el Reglamento 1151/2012 y las disposiciones relativas a las indicaciones geográficas previstas en el Reglamento 1308/2013 tampoco se aplican a los productos vitivinícolas aromatizados, considerándose éstos importantes para los consumidores, los productores y el sector agrícola de la Unión, se ha visto conveniente establecer unas normas específicas en materia de protección de las indicaciones geográficas de estos productos. Concretamente nos referimos a vinos aromatizados, bebidas aromatizadas a base de vino y cócteles aromatizados de productos vitivinícolas.

A los efectos del Reglamento (UE) No 251/2014 del Parlamente Europeo y del Consejo, de 26 de febrero de 2014, sobre la definición, descripción, presentación, etiquetado y protección de las indicaciones geográficas de los productos vitivinícolas aromatizados, se entiende por «indicación geográfica», una denominación que identifica a un producto vitivinícola aromatizado como procedente de una región, un lugar determinado o un país en que una determinada calidad, renombre, u otras características de ese producto, es en esencia atribuible a su origen geográfico ${ }^{16}$. Los productos vitivinícolas aromatizados se elaborarán de acuerdo con los requisitos, restricciones y designaciones establecidos en los anexos I y II del citado Reglamento.

Resulta digno de destacar que en estas dos normativas no se hace referencia al término denominación de origen, aglutinando todas las designaciones geográficas de calidad en torno a la única figura de indicación geográfica.

${ }^{16}$ Artículo 2. 3) del Reglamento 251/2014. 


\section{CONCLUSIONES}

La importancia de la protección de las referencias geográficas de calidad de los productos agroalimentarios, como motor de desarrollo de las economías territoriales, queda patente ante la existencia de varios regímenes jurídicos que a nivel europeo tratan de regularlas.

La existencia de una específica y extensa regulación para los productos vinícolas evidencia la importancia que éstos registran para numerosos países europeos, que ven precisa una regulación aparte para esta clase de productos, fundamentales para el desarrollo económico de determinadas regiones, principalmente del área mediterránea.

La existencia de una regulación a nivel de la Unión Europea sobre este tipo de referencias geográficas de calidad, deja en un segundo plano las normativas estatales y regionales, que deben amparar sus propias figuras de protección en torno a las figuras generales de Denominación de Origen Protegida e Indicación Geográfica Protegida, pudiendo mantener sus específicas denominaciones bajo las categorías de Términos Tradicionales (caso del vino) o Especialidades Tradicionales Garantizadas (productos no vinícolas), subsumidas en algunas de las categorías de calidad generales, DOP o IGP. La existencia de numerosos términos o especialidades tradicionales, principalmente en el sector vinícola, dificulta la comprensión por parte del consumidor de los mismos, sobre todo cuando se trata de productos procedentes de Estados miembros diferentes al del propio consumidor, que deberá ejercer una importante labor de investigación para reconocer el alcance de estos términos de calidad de segundo nivel. Asimismo, al igual que la Comisión Europea ${ }^{17}$, compartimos nuestra preocupación ante la creación y admisión de Términos de Calidad Facultativos para designar productos agroalimentarios no vinícolas, como sistemas de calidad de segundo nivel, que, a nuestro juicio, aumentaría aún más el grado de confusión ya existente en el consumidor sobre referencias geográficas de calidad de los productos.

Por otro lado, la regulación diferenciada en atención a la clase de producto de que se trate dificulta la comprensión por parte del consumidor de los términos geográficos de calidad, dificultad que se ve incrementada con la adopción de dos figuras de protección: Denominación de Origen Protegida (DOP) e Indicación Geográfica Protegida (IGP), con igual nivel de protección y mismo tratamiento jurídico. El consumidor no llega a distinguir ambas figuras, pudiendo incluso provocar conflictos entre los productores para la obtención de una u otra, así como crear problemas de concurrencia de ambas figuras en un mismo territorio para designar una misma clase de productos. Sería conveniente, a nuestro juicio,

${ }^{17}$ Ver conclusiones del Informe de la Comisión al Parlamento Europeo y al Consejo sobre la conveniencia del término de calidad facultativo "producto de la agricultura insular». Bruselas, 16.12.2013. $\operatorname{COM(2013)~} 888$ final. 
aglutinar en una sola figura de protección todas las referencias geográficas de calidad de los productos agroalimentarios, bajo el término Indicación Geográfica Protegida (IGP), al menos en los productos no vinícolas, donde el término denominación de origen no se encuentra tan arraigado.

Finalmente, la regulación europea de las referencias geográficas de calidad de los productos no debería circunscribirse únicamente a los productos agroalimentarios, siendo necesario extender la protección a otra clase de productos, como así se viene reconociendo en la normativa internacional sobre propiedad intelectual y cuya procedencia está siendo estudiada en estos momentos por la Comisión Europea para su presentación ante el Parlamento y Consejo Europeos. Eso sí, a nuestro parecer, debería establecerse una única figura de protección, la Indicación Geográfica Protegida (IGP) y no caer de nuevo, a nuestro juicio, en el error de una terminología dual, que lo único que redunda es en una indeseada confusión del consumidor y en una polémica entre productores.

\section{BIBLIOGRAFÍA}

Albisinni F., Wines, [en:] European Food Law, Coord. L. Costato, F. Albisinni, Milán 2012.

Botana Agra M.J., Las Denominaciones de Origen, [en:] Tratado de Derecho Mercantil, Vol. 2: Las Denominaciones de Origen, Coord. G. Jiménez Sánchez, Barcelona 2001.

Documento SCT/8/4, de 2 de abril de 2002, del Comité Permanente sobre el Derecho de Marcas, Dibujos y Modelos industriales e Indicaciones Geográficas de la OMPI, www.wipo.int [accesso: 10.03.2017].

Fernández-Nóvoa C., La protección internacional de las denominaciones geográficas de los productos, Madrid 1970.

Gómez Lozano M.M., Denominaciones de Origen y otras Indicaciones Geográficas, Navarra 2004.

Guillem Carrau J., Denominaciones Geográficas de Calidad. Estudio de su reconocimiento y protección en la OMC, la UE y el Derecho español, Valencia 2008.

López Benítez M., Del Estatuto del vino a las leyes del vino: un panorama actual y de futuro de la ordenación vitivinícola en España, Madrid, 2004.

López Benítez M., El momento actual de las denominaciones geográficas vitivinicolas, [en:] Derechos sociales y estatutos de autonomía, denominaciones de origen, nuevo estatuto del PDI universitario: Actas del IV Congreso de la Asociación Española de Profesores de Derecho Administrativo, Coord. J.L. Martínez López-Muñiz, Valladolid 2009.

López Benítez M., Las denominaciones de origen, Barcelona 1996.

Maroño Gargallo M.M., La protección jurídica de las denominaciones de origen en los derechos español y comunitario, Madrid 2002.

Martínez Gutiérrez A., La designación del origen geográfico en la comercialización de los aceites de oliva. Especial referencia a la viabilidad jurídica de las marcas oleícolas de carácter geográfico, Málaga 2011.

Martínez Gutiérrez A., La tutela comunitaria de las denominaciones geográficas. Conflictos con otros signos distintivos, Barcelona 2008.

Martínez Gutiérrez A., Nuevos títulos de protección de carácter comunitario para los vinos de calidad, Navarra 2011. 
Pobrane z czasopisma Studia Iuridica Lublinensia http://studiaiuridica.umes.pl Data: 26/04/2023 14:11:43

Organización Mundial de la Propiedad Intelectual, Principios Básicos de la Propiedad Industrial. Publicación de la OMPI nº 895(S), www.wipo.int [accesso: 10.03.2017].

\section{STRESZCZENIE}

Obrót produktami rolno-żywnościowymi chronionymi za pomocą oznaczeń geograficznych symptomatycznie wzrasta m.in. ze względu na przypisywanie im przez konsumentów wysokich cech jakościowych związanych z miejscem ich wytworzenia. W związku z powyższym przyczyniło się to do rozwoju gospodarczego tych obszarów, co - jak należy przypuszczać - nie miałoby miejsca, gdyby nie opatrzenie wspomnianych dóbr chronionymi oznaczeniami geograficznymi. Z tej właśnie przyczyny kwestia form ochronnych produktów rolno-żywnościowych stanowi niezwykle ważny element dla poszczególnych rządów krajowych i regionalnych, prowadząc tym samym do rozwoju systemu prawnego związanego z tą problematyką. Dlatego cel niniejszego artykułu stanowi analiza porządku prawnego Unii Europejskiej regulującego system ochrony produktów rolno-żywnościowych za pomocą oznaczeń geograficznych odnoszących się do jakości.

Słowa kluczowe: własność przemysłowa; Chronione Oznaczenia Geograficzne; Chronione Nazwy Pochodzenia; produkty rolno-żywnościowe; produkty winiarskie; rozwój obszarów wiejskich 